\title{
A SIMPLE PROOF OF THE GENERALIZED LEIBNIZ RULE ON BOUNDED EUCLIDEAN DOMAINS
}

\author{
QUOC-HUNG NGUYEN, YANNICK SIRE, AND JUAN-LUIS VÁZQUEZ
}

\begin{abstract}
This note is devoted to a simple proof of the generalized Leibniz rule in bounded domains. The operators under consideration are the so-called spectral Laplacian and the restricted Laplacian. Equations involving such operators have been lately considered by Constantin and Ignatova in the framework of the SQG equation [CI16] in bounded domains, and by two of the authors [NV18] in the framework of the porous medium with nonlocal pressure in bounded domains. We will use the estimates in this work in a forthcoming paper on the study of Porous Medium Equations with pressure given by Riesz-type potentials.
\end{abstract}

\section{Contents}

1. Introduction 1

2. Proof of Theorem 1.1: the case of the spectral Laplacian 5

3. Proof of Theorem $1.2 \quad 8$

Appendix $\quad 9$

References 12

\section{INTRODUCTION}

Commutator estimates are instrumental in the study of PDEs. Recently, several models arising in Fluid dynamics for instance, involve Fourier multipliers in $\mathbb{R}^{n}$ of the type $|\xi|^{2 \alpha}$ where $\alpha>0$ is real. The case of bounded domains, hence outside of the theory of multipliers, has been considered e.g. in [CI16].

The purpose of the present note is to provide a very simple proof of a commutator estimate in the setting of operators defined on bounded domains. In such a case, one cannot rely on the classical theory of para-differential operators, and one has to use other types of tools to derive the estimates. Heat kernel methods are very useful in this setting since they require very little a priori assumptions on the domain. Here we consider another approach, much more general somehow, relying on harmonic extensions as in [CS07, ST10], and inspired by [NV18, Lemma 2].

Fractional operators on bounded domains. In the following we consider $\Omega$ a bounded smooth domain of $\mathbb{R}^{d}$ with $d \geq 1$. We first list the two nonlocal operators that serve as motivation.

The Spectral Laplacian. Consider the eigensystem $\left(\varphi_{j}, \lambda_{j}\right)$ for the Dirichlet Laplacian acting in $\Omega$, namely $-\Delta \varphi_{j}=\lambda_{j} \varphi_{j}$ with homogeneous Dirichlet boundary conditions. It is well known that $0<\lambda_{1} \leq \ldots \leq \lambda_{j} \leq \ldots$, that $\lambda_{j} \asymp j^{2 / d}$, and that $-\Delta$ is a positive self-adjoint 
operator in $L^{2}(\Omega)$ with domain $D(-\Delta)=H^{2}(\Omega) \cap H_{0}^{1}(\Omega)$. The ground state $\varphi_{1}$ is positive and $\varphi_{1}(x) \asymp d(x)$ for all $x \in \Omega$, where $d(x)$ denotes distance to boundary. For all $0<\alpha<1$ we define the spectral fractional Laplacian, $(-\Delta)_{s p}^{\alpha}$, by

$$
(-\Delta)_{s p}^{\alpha} u=\sum_{j=1}^{\infty} \lambda_{j}^{\alpha} u_{j} \varphi_{j}, \quad u_{j}=\int_{\Omega} u(x) \varphi_{j}(x) d x
$$

The Restricted Laplacian. One can define a fractional Laplacian operator by using the integral representation in terms of hypersingular kernels (for instance for locally $C^{1,1}$ functions and bounded over $\mathbb{R}^{d}$ )

$$
\left(-\Delta_{\mathbb{R}^{d}}\right)^{\alpha} g(x)=c_{d, s} \text { P.V. } \int_{\mathbb{R}^{d}} \frac{g(x)-g(z)}{|x-z|^{d+2 \alpha}} d z,
$$

where $c_{d, \alpha}>0$ is a normalization constant. In this case we materialize the zero Dirichlet condition by restricting the operator to act only on functions that are zero outside $\Omega$. We will call the operator defined in such a way the restricted fractional Laplacian.

It is a well-known fact that the two previously defined operators are different. This is easily recognized by simple properties, like the fact that they have different spectral sequences, or the fact that the solutions of the Dirichlet Problem have different boundary behaviour and a different Green function, see [BSV15, BV16, $\mathrm{CdPF}^{+} 17, \mathrm{CS} 05, \mathrm{MN} 14$, SV14].

Commutator results and the idea of the proof. Our main results are the following. The first deals with our main contribution, i. e., a Leibniz rule for the operator defined above as the spectral Laplacian.

Theorem 1.1. Let $0<\beta \leq \alpha<\frac{1}{2}$ and $f, g, h \in C_{0}^{\infty}(\Omega)$. Let $(-\Delta)_{s p}^{\alpha}$ denote the spectral Laplacian acting in a smooth bounded domain $\Omega$ in $\mathbb{R}^{d}, d \geq 1$. There exists $C>0$ such that

$$
\begin{aligned}
& \left\|(-\Delta)_{s p}^{\alpha}(g h)-g(-\Delta)_{s p}^{\alpha} h-h(-\Delta)_{s p}^{\alpha} g\right\|_{L^{2}(\Omega)} \\
& \leq C\left\|(-\Delta)_{s p}^{\frac{\beta}{2}} h\right\|_{L^{2}(\Omega)}\|g\|_{L^{\infty}(\Omega)}^{\frac{\beta}{2 \alpha}}\left\|(-\Delta)_{s p}^{\alpha} g\right\|_{L^{\infty}(\Omega)}^{\frac{2 \alpha-\beta}{2 \alpha}} .
\end{aligned}
$$

As a matter of fact, the proof of Theorem 1.1 is an adaptation of a strategy that can be easily implemented also in the case of the fractional Laplacian defined in the whole of $\mathbb{R}^{d}$. We state then the following

Theorem 1.2. Let $0<\beta \leq \alpha<\frac{1}{2}$ and $f, g, h \in C_{0}^{\infty}\left(\mathbb{R}^{d}\right)$ for $d \geq 1$. Let $(-\Delta)^{\alpha}$ denote the operator with Fourier multiplier $|\xi|^{2 \alpha}$ in $\mathbb{R}^{d}$ with $d \geq 1$. There exists $C>0$ such that

$$
\begin{aligned}
& \left\|(-\Delta)^{\alpha}(g h)-g(-\Delta)^{\alpha} h-h(-\Delta)^{\alpha} g\right\|_{L^{2}\left(\mathbb{R}^{d}\right)} \\
& \leq C\left\|(-\Delta)^{\frac{\beta}{2}} h\right\|_{L^{2}\left(\mathbb{R}^{d}\right)}\|g\|_{L^{\infty}\left(\mathbb{R}^{d}\right)}^{\frac{\beta}{2 \alpha}}\left\|(-\Delta)^{\alpha} g\right\|_{L^{\infty}\left(\mathbb{R}^{d}\right)}^{\frac{2 \alpha-\beta}{2 \alpha}} .
\end{aligned}
$$

The previous theorems whenever $(-\Delta)^{\alpha}$ is the standard fractional Laplacian with Fourier symbol $|\xi|^{2 \alpha}$ are known as a version of a commutator estimate by Kenig, Ponce and Vega [KPV93] (see also the famous Kato-Ponce estimate [KP88]). More precisely, the KenigPonce-Vega estimate in $\mathbb{R}^{d}$ writes

$$
\left\|(-\Delta)^{\alpha}(g h)-g(-\Delta)^{\alpha} h-h(-\Delta)^{\alpha} g\right\|_{L^{p}} \lesssim\left\|(-\Delta)^{\alpha_{1}} g\right\|_{L^{p_{1}}}\left\|(-\Delta)^{\alpha_{2}} h\right\|_{L^{p_{2}}}
$$

whenever $\alpha=\alpha_{1}+\alpha_{2}, 0<\alpha, \alpha_{1}, \alpha_{2}<1 / 2, \frac{1}{p}=\frac{1}{p_{1}}+\frac{1}{p_{2}}, 1<p, p_{1}, p_{2}<\infty$. 
We would like also to point out that recently in [Li19] D. Li obtained for the first time a general Leibniz rule for all fractional Laplacian operators $(-\Delta)^{\alpha}$ including the difficult end-point cases, solving in particular a conjecture by Kato and Ponce. Since we are interested for our applications in the $L^{2}$ estimate, and since the proof is very simple and straightforward in this case, we focus only on these particular exponents. It must be said that the full estimates for the left-hand side in our main theorem in other Lebesgue spaces, which is important for other applications, can be obtained following the same type of strategy. However, in this latter case, instead of using simple integration by parts followed by Hölder and/or Hardy inequality, one needs to invoke Caldéron-Zygmund inequalities and interpolation theory for instance. A full account on such estimates in the Euclidean space using Littlewood-Paley theory can be found in the mentioned paper by Li [Li19].

Remark 1.1. It will be clear from the proof that the case $\beta=0$ is allowed. This is actually a version of Theorem 1.2 in [Li19]. In this later, the author managed even, invoking Coifman-Meyer theorem for instance, to replace the $L^{\infty}$ norm by the weaker $B M O$ norm.

The proof of Theorems 1.1 and 1.2 is based on the following idea: owing to [CS07, ST10], powers of the Laplacian can be realized as the Dirichlet-to-Neumann operator of a suitable extension. Such extensions are also valid for the spectral and restricted Laplacian, as described more precisely below. This allows to use the elliptic PDE satisfied in the extended domain via integration by parts to get the desired cancellation. A unified theory for several non-trivial sharp commutator estimates based on this idea has also been extensively studied by Lenzmann and Schikorra [LS20] (see also [BSS20b] for a geometric version of such extension). Our proof is inspired also by the one of [NV18, Lemma 2]. It should be noticed that one can find higher order Kato-Ponce and Leibniz commutator estimates in [Li19]. Since we make use of a PDE technique via an extension, the case of higher order operators $\alpha \geq 1 / 2$ needs to be treated differently. Even for $\alpha \geq 1$, it requires the extension formulae developed in [CY17] and a rather technical adaptation of the present simple argument. This is under investigation by Dong Li and the second author [LS21].

We would like to emphasize that in Theorem 1.2 the norms are taken in the whole space $\mathbb{R}^{d}$. If one considers the restricted Laplacian, one could ask if one can take the norms in the domain $\Omega$ where the functions $g$ and $h$ are supported: the analogue of the estimate (1.3) whenever norms are taken in $\Omega$ is an open problem and we conjecture that in general such an estimate does not hold. The reason why the latter seems to be unlikely to hold is probably due to a combination of the tail effects of the restricted Laplacian and the boundary behaviour of the functions (which relies crucially on the parameter $\alpha$ ). However, we conjecture that there should be a natural replacement for the Leibniz rule in this case. In the appendix we provide two estimates supporting our conjecture.

We recall that the fractional Laplacian in Theorem 1.4 whenever the functions are supported in $\Omega \subset \mathbb{R}^{d}$ is not the so-called regional fractional Laplacian which is defined by

$$
(-\Delta)_{\Omega}^{\alpha} g:=\int_{\Omega} \frac{g(x)-g(y)}{|x-y|^{d+2 \alpha}} d y \text { in } \Omega .
$$

These three different operators play an important role in the theory of Lévy processes (see e.g. the recent papers [KSV20, KSV19]). However, we are not aware of any kind of replacement of Theorem 1.4 in the case of the censored Laplacian, and as a consequence we cannot run the same argument to prove a generalized Leibniz rule. Furthermore, the censored Laplacian is not a pseudo-differential operator in the classical sense so that standard techniques do not seem to apply successfully. 
Review of the extension results. We now state the two versions of the extension problem we need to implement the strategy of the proof of our main result. We state first the main result in [ST10], Theorem 1.1 (see also[CT10, CDDS11]), which allows us to deal with the Spectral Laplacian.

Theorem 1.3. Let $d \geq 1$ and $\alpha \in(0,1)$. Consider $g \in C_{0}^{\infty}(\Omega)$; then the function defined by

$$
u(x, y)=\frac{y^{2 \alpha}}{4^{\alpha} \Gamma(\alpha)} \int_{0}^{\infty} e^{-\frac{y^{2}}{4 t}} e^{t \Delta_{s p}} g(x) \frac{d t}{t^{1+\alpha}}=\frac{1}{\Gamma(\alpha)} \int_{0}^{\infty} e^{-\frac{y^{2}}{4 t}} e^{t \Delta_{s p}}\left[(-\Delta)_{s p}^{\alpha} g\right](x) \frac{d t}{t^{1-\alpha}}
$$

solves in the weak sense the boundary value problem

$$
\begin{cases}\operatorname{div}\left(y^{1-2 \alpha} \nabla u\right)=0 & \text { in } \Omega \times(0, \infty), \\ u=0 & \text { on } \partial \Omega \times(0, \infty), \\ u(x, 0)=g(x) & \text { in } \Omega .\end{cases}
$$

Furthermore, one has pointwise

$$
-\lim _{y \rightarrow 0^{+}} y^{1-2 \alpha} u_{y}(x, y)=(-\Delta)_{s p}^{\alpha} g(x) .
$$

Remark 1.2. Theorem 1.3 holds in a much more general setting than the one used here. In particular, it opens the way to get a wide class of commutator estimates in non-Euclidean frameworks (see [BSS20b, BSS20a]).

Secondly, we state the one useful for the Fractional Laplacian, due to [CS07].

Theorem 1.4. Let $d \geq 1, \alpha \in(0,1)$ and $g \in C_{0}^{\infty}\left(\mathbb{R}^{d}\right)$. Denote by $P_{\alpha}$ is the Poisson kernel of $\operatorname{div}\left(y^{1-2 \alpha} \nabla \cdot\right)$ in $\mathbb{R}_{+}^{d+1}$, i.e.

The function defined by

$$
P_{\alpha}(x, y)=\frac{y^{2 \alpha}}{\left(|x|^{2}+y^{2}\right)^{\frac{d+2 \alpha}{2}}} .
$$

$$
u(x, y)=\int_{\mathbb{R}^{d}} P_{\alpha}(x-t, y) g(t) d t
$$

solves in the weak sense the boundary value problem

$$
\begin{cases}\operatorname{div}\left(y^{1-2 \alpha} \nabla u\right)=0 & \text { in } \mathbb{R}_{+}^{d+1}, \\ u(x, 0)=g(x) & \text { on } \partial \mathbb{R}_{+}^{d+1} \sim \mathbb{R}^{d} .\end{cases}
$$

Furthermore, one has pointwise

$$
-\lim _{y \rightarrow 0^{+}} y^{1-2 \alpha} u_{y}(x, y)=(-\Delta)^{\alpha} g(x) .
$$

Remark 1.3. As noticed in [ST10], by the very construction of $u$ in the previous theorems, the solution itself enjoys decay properties at $\infty$. In particular, this justifies the computations in the following sections.

Comments. A classical technique to prove commutator estimates is to use LittlewoodPaley decomposition and Coifman-Meyer estimates combined with other deep harmonic analysis tools. We adopt here a different strategy based on the unified approach by the first two authors and then Lenzmann and Schikorra previously mentioned; that approach does not require at all in our case any deep result on boundedness of multipliers. It allows in particular to get sharp estimates in much more general frameworks, at least for operators 
given by powers of the second order operators, than the paraproduct technique. Indeed, a major drawback of using paraproducts is their intrinsic Euclidean nature. However, Bernicot and Frey (see e.g. [Fre13] and references therein) developed in the last decade a theory of para-differential calculus on geometric settings where the standard Fourier analysis is not available. However, for many PDE applications, such a theory is rather involved and, as the proof of [NV18, Lemma 2] and the unified approach developed by Lenzmann and Schikorra (and ours here) shows, one can get in many cases a much simpler approach.

Notations. in $\mathbb{R}^{d+1}=\left\{(x, y), x \in \mathbb{R}^{d}, y \in \mathbb{R}\right\}$, we will denote the classical differential operators $\nabla=\left(\nabla_{x}, \partial_{y}\right)$, div $=\operatorname{div}_{x}+\partial_{y}$ and $\Delta=\Delta_{x}+\partial_{y y}$ with the obvious meanings.

\section{Proof of Theorem 1.1: the case of the spectral Laplacian}

Harmonic extensions. (i) We define the following three quantities which play the important role in the proof of the commutator estimate. Let $U, V, W$ be the solutions given by Theorem 1.3 of the boundary value problems:

$$
\begin{aligned}
& \begin{cases}\operatorname{div}\left(y^{1-2 \alpha} \nabla U\right)=0 & \text { in } \Omega \times(0, \infty), \\
U=0 & \text { on } \partial \Omega \times(0, \infty), \\
U(x, 0)=g(x) & \text { in } \Omega,\end{cases} \\
& \begin{cases}\operatorname{div}\left(y^{1-2 \alpha} \nabla V\right)=0 & \text { in } \Omega \times(0, \infty), \\
V=0 & \text { on } \partial \Omega \times(0, \infty), \\
V(x, 0)=h(x) & \text { in } \Omega,\end{cases} \\
& \begin{cases}\operatorname{div}\left(y^{1-2 \alpha} \nabla W\right)=0 & \text { in } \Omega \times(0, \infty), \\
W=0 & \text { on } \partial \Omega \times(0, \infty), \\
W(x, 0)=g(x) h(x) & \text { in } \Omega\end{cases}
\end{aligned}
$$

We introduce the new function $Z=W-U V$. By construction we have

$$
-\lim _{y \rightarrow 0^{+}} y^{1-2 \alpha} Z_{y}(x, y)=\left[(-\Delta)_{s p}^{\alpha}(g h)(x)-g(x)(-\Delta)_{s p}^{\alpha}(h)(x)-h(x)(-\Delta)_{s p}^{\alpha}(g)(x)\right] .
$$

$Z$ satisfies a state equation and boundary data:

$$
\begin{cases}\operatorname{div}\left(y^{1-2 \alpha} \nabla Z\right)=-2 y^{1-2 \alpha} \nabla U \cdot \nabla V & \text { in } \Omega \times(0, \infty), \\ Z=0 & \text { on } \partial \Omega \times(0, \infty), \\ Z(x, 0)=0 & \text { in } \Omega .\end{cases}
$$

Multiplying (2.4) by $\varphi=y^{-2 \alpha} Z$ and integrating by parts leads

$$
\begin{aligned}
\int_{0}^{\infty} & \int_{\Omega} y^{1-2 \alpha} Z_{y} \partial_{y}\left(y^{-2 \alpha} Z\right) d x d y+\int_{\Omega} \lim _{y \rightarrow 0}\left[y^{1-2 \alpha} Z_{y} y^{-2 \alpha} Z\right] d x \\
+ & \int_{0}^{\infty} \int_{\Omega} y\left|\nabla_{x}\left(y^{-2 \alpha} Z\right)\right|^{2} d x d y=2 \int_{0}^{\infty} \int_{\Omega} y^{1-2 \alpha} \nabla U \cdot \nabla V y^{-2 \alpha} Z d x d y .
\end{aligned}
$$

Since $y^{1-2 \alpha} Z_{y}=y \partial_{y}\left(y^{-2 \alpha} Z\right)+2 \alpha y^{-2 \alpha} Z$

$$
\begin{aligned}
\int_{0}^{\infty} \int_{\Omega} y^{1-2 \alpha} Z_{y} \partial_{y}\left(y^{-2 \alpha} Z\right) d x d y & =\int_{0}^{\infty} \int_{\Omega} y\left|\partial_{y}\left(y^{-2 \alpha} Z\right)\right|^{2} d x d y+\alpha \int_{0}^{\infty} \int_{\Omega} \partial_{y}\left[\left(y^{-2 \alpha} Z\right)^{2}\right] \\
& =\int_{0}^{\infty} \int_{\Omega} y\left|\partial_{y}\left(y^{-2 \alpha} Z\right)\right|^{2} d x d y-\alpha \int_{\Omega} \lim _{y \rightarrow 0}\left|y^{-2 \alpha} Z\right|^{2} d x .
\end{aligned}
$$


Thanks to $Z(x, 0)=0$, one has

$$
\lim _{y \rightarrow 0} y^{-2 \alpha} Z=\frac{1}{2 \alpha} \lim _{y \rightarrow 0} y^{1-2 \alpha} Z_{y} \text { a.e in } \Omega .
$$

Combining this with (2.6) and (2.5) we arrive at

$$
\begin{aligned}
& \frac{1}{4 \alpha} \int_{\Omega}\left|\lim _{y \rightarrow 0} y^{1-2 \alpha} Z_{y}(x, y)\right|^{2} d x+\int_{0}^{\infty} \int_{\Omega} y\left|\nabla_{x}\left(y^{-2 \alpha} Z\right)\right|^{2} d x d y \\
& \quad=2 \int_{0}^{\infty} \int_{\Omega} y^{1-2 \alpha} \nabla U \cdot \nabla V y^{-2 \alpha} Z d x d y .
\end{aligned}
$$

Our goal is to estimate the first term in the left-hand side in order to get the commutator estimate of Theorem 1.1. Discarding the second term and using Hölder's inequality on the last term, we get

$$
\begin{aligned}
& \int_{\Omega}\left|\lim _{y \rightarrow 0} y^{1-2 \alpha} Z_{y}(x, y)\right|^{2} d x \\
& \leq\left. C|| y^{1-2 \alpha}|\nabla U|\right|_{L^{\infty}(\Omega \times(0, \infty))} \int_{0}^{\infty} \int_{\Omega}|\nabla V| y^{-2 \alpha}|Z| d x d y \\
& \leq C\left(\int_{0}^{\infty} \int_{\Omega} y^{1-2 \beta}|\nabla V|^{2} d x d y\right)^{1 / 2}\left(\int_{0}^{\infty} \int_{\Omega} y^{-1-2(2 \alpha-\beta)}|Z|^{2} d x d y\right)^{1 / 2} \\
& \quad \times\left\|y^{1-2 \alpha}|\nabla U|\right\|_{L^{\infty}((0, \infty) \times \Omega)}
\end{aligned}
$$

for $0<\beta \leq \alpha<\frac{1}{2}$. At this point, it is enough to show the following three inequalities:

$$
\begin{gathered}
\int_{0}^{\infty} \int_{\Omega} y^{1-2 \beta}|\nabla V|^{2} d x d y \leq C \int_{\Omega}\left|(-\Delta)_{s p}^{\frac{\beta}{2}} h\right|^{2} d x, \\
\int_{0}^{\infty} \int_{\Omega} y^{-1-2 \alpha}|Z|^{2} d x d y \leq C\left\|(-\Delta)_{s p}^{\frac{\beta}{2}}(h)\right\|_{L^{2}(\Omega)}^{2}\left\|\left.g\right|_{L^{\infty}(\Omega)} ^{\frac{2 \beta}{\alpha}}\right\|(-\Delta)_{s p}^{\alpha}(g) \|_{L^{\infty}(\Omega)}^{\frac{2(\alpha-\beta)}{\alpha}},
\end{gathered}
$$

and finally

$$
\left\|y^{1-2 \alpha}|\nabla U|\right\|_{L^{\infty}(\Omega \times(0, \infty))} \leq C||(-\Delta)_{s p}^{\alpha}(g) \|_{L^{\infty}(\Omega)} .
$$

Proof of (2.8): Let $\phi$ be the unique solution of

$$
\begin{cases}\operatorname{div}\left(y^{1-2 \beta} \nabla \phi\right)=0 & \text { in } \Omega \times(0, \infty), \\ \phi=0 & \text { on } \partial \Omega \times(0, \infty), \\ \phi(x, 0)=h(x) & \text { in } \Omega .\end{cases}
$$

It is enough to check that

$$
\int_{0}^{\infty} \int_{\Omega} y^{1-2 \beta}|\nabla V|^{2} \leq C \int_{0}^{\infty} \int_{\Omega} y^{1-2 \beta}|\nabla \phi|^{2}
$$

since $\int_{\Omega}\left|(-\Delta)_{s p}^{\frac{\beta}{2}} h\right|^{2} d x=c \int_{0}^{\infty} \int_{\Omega} y^{1-2 \beta}|\nabla \phi|^{2}$ by the very definition of the extension and for a universal constant $c$ (see [ST10]). Since

$$
\operatorname{div}\left(y^{1-2 \alpha} \nabla \phi\right)=2(\beta-\alpha) y^{-2 \alpha} \phi_{y}
$$

the function $\bar{\phi}=\phi-V$ satisfies

$$
\begin{cases}\operatorname{div}\left(y^{1-2 \alpha} \nabla \bar{\phi}\right)=2(\beta-\alpha) y^{-2 \alpha} \phi_{y} & \text { in } \Omega \times(0, \infty), \\ \bar{\phi}=0 & \text { on } \partial \Omega \times(0, \infty), \\ \bar{\phi}(x, 0)=0 & \text { in } \Omega\end{cases}
$$


Choosing $y^{2(\alpha-\beta)} \bar{\phi}$ as a test function for the above equation, one has

$$
\int_{0}^{\infty} \int_{\Omega} y^{1-2 \beta}|\nabla \bar{\phi}|^{2}+2(\alpha-\beta) y^{-2 \beta} \bar{\phi}_{y} \bar{\phi}=2(\alpha-\beta) \int_{0}^{\infty} \int_{\Omega} y^{-2 \beta} \phi_{y} \bar{\phi} .
$$

Notice that

$$
2(\alpha-\beta) \int_{0}^{\infty} \int_{\Omega} y^{-2 \beta} \bar{\phi}_{y} \bar{\phi} d x d y=2(\alpha-\beta) \beta \int_{0}^{\infty} \int_{\Omega} y^{-1-2 \beta}|\bar{\phi}|^{2} \geq 0 .
$$

Furthermore, using Hölder's and Young's inequality, one gets for some $\varepsilon>0$ to be chosen

$$
\begin{aligned}
& \int_{0}^{\infty} \int_{\Omega} y^{1-2 \beta}|\nabla \bar{\phi}|^{2}+2(\alpha-\beta) \beta \int_{0}^{\infty} \int_{\Omega} y^{-1-2 \beta}|\bar{\phi}|^{2} \\
& \leq(\alpha-\beta)\left(\varepsilon \int_{0}^{\infty} \int_{\Omega} y^{-1-2 \beta}|\bar{\phi}|^{2}+\frac{1}{\varepsilon} \int_{0}^{\infty} \int_{\Omega} y^{1-2 \beta}|\nabla \phi|^{2}\right) .
\end{aligned}
$$

Choosing $\varepsilon$ small enough and discarding the nonnegative term in the LHS, this implies that

$$
\int_{0}^{\infty} \int_{\Omega} y^{1-2 \beta}|\nabla \bar{\phi}|^{2} \leq C \int_{0}^{\infty} \int_{\Omega} y^{1-2 \beta}|\nabla \phi|^{2} d x d y
$$

which implies (2.12) recalling that $\bar{\phi}=\phi-V$.

Proof of (2.10): By standard regularity theory for the heat kernel ( see e.g. [Dav90]): for all $\phi \in L^{\infty}$ and all $x \in \Omega$

$$
\left|\nabla_{x} e^{t \Delta_{s p}} \phi(x)\right| \leq C \frac{1}{\sqrt{t}} \min \left\{1, \frac{1}{t^{\frac{d}{2}}}\right\}\|\phi\|_{L^{\infty}(\Omega)},\left|e^{t \Delta_{s p}} \phi(x)\right| \leq C \min \left\{1, \frac{1}{t^{\frac{d}{2}}}\right\}\|\phi\|_{L^{\infty}(\Omega)} .
$$

Using these estimates in formulas (1.6) (and obvious changes of variables), one gets

$$
\begin{aligned}
& |y|^{1-2 \alpha}|\nabla U(x, y)| \leq C|y|^{1-2 \alpha} \int_{0}^{\infty}\left(\frac{1}{\sqrt{t}}+\frac{|y|}{t}\right) e^{-\frac{y^{2}}{4 t}} \frac{d t}{t^{1-\alpha}}\left\|(-\Delta)_{s p}^{\alpha} g\right\|_{L^{\infty}(\Omega)}, \\
& |y||\nabla U(x, y)| \leq C|y|^{1+2 \alpha} \int_{0}^{\infty}\left(\frac{1}{|y|}+\frac{|y|}{t}+\frac{1}{\sqrt{t}}\right) e^{-\frac{y^{2}}{4 t}} \frac{d t}{t^{1+\alpha}}\|g\|_{L^{\infty}(\Omega)},
\end{aligned}
$$

which implies (2.10) and

$$
\|y|\nabla U|\|_{L^{\infty}(\Omega \times(0, \infty))} \leq C\|g\|_{L^{\infty}(\Omega)} .
$$

Proof of (2.9): We choose $\varphi=y^{-2(\alpha-\beta)} Z$ as a test function for (2.4)

$$
\begin{aligned}
& \int_{0}^{\infty} \int_{\Omega} y^{1-2(2 \alpha-\beta)}|\nabla Z|^{2} d x d y-2(\alpha-\beta) \int_{0}^{\infty} \int_{\Omega} y^{-2(2 \alpha-\beta)} Z_{y} Z d x d y \\
& =2 \int_{0}^{\infty} \int_{\Omega} y^{1-2(2 \alpha-\beta)} \nabla U \cdot \nabla V Z d x d y .
\end{aligned}
$$

Using the following Hardy's inequality (see [Zyg59], p.20)

$$
\int_{0}^{\infty} y^{1-2(2 \alpha-\beta)}\left|Z_{y}\right|^{2} d y \geq(2 \alpha-\beta)^{2} \int_{0}^{\infty} y^{-1-2(2 \alpha-\beta)}|Z|^{2} d y
$$

and the fact that

$$
-2(\alpha-\beta) \int_{0}^{\infty} \int_{\Omega} y^{-2(2 \alpha-\beta)} Z_{y} Z d x d y=-2(\alpha-\beta)(2 \alpha-\beta) \int_{0}^{\infty} \int_{\Omega} y^{-1-2(2 \alpha-\beta)}|Z|^{2} d x d y,
$$


yields

$$
\begin{aligned}
& \frac{\beta}{2 \alpha-\beta} \int_{0}^{\infty} \int_{\Omega} y^{1-2(2 \alpha-\beta)}|\nabla Z|^{2} d x d y \\
& \quad \leq \operatorname{LHS}(2.15)=2 \int_{0}^{\infty} \int_{\Omega} y^{1-2(2 \alpha-\beta)} \nabla U \cdot \nabla V Z d x d y .
\end{aligned}
$$

Using Hölder's inequality (together with Young's inequality) and (2.17), we get

$$
\begin{aligned}
& \int_{0}^{\infty} \int_{\Omega} y^{1-2(2 \alpha-\beta)}|\nabla Z|^{2} \leq C \int_{0}^{\infty} \int_{\Omega} y^{3-2(2 \alpha-\beta)}|\nabla V|^{2}|\nabla U|^{2} \\
& \leq C\left\|y^{1-2(\alpha-\beta)}|\nabla U|\right\|_{L^{\infty}(\Omega \times(0, \infty))}^{2} \int_{0}^{\infty} \int_{\Omega} y^{1-2 \beta}|\nabla V|^{2} \\
& \stackrel{(2.8)}{\leq} C\left\|(-\Delta)^{\frac{\beta}{2}}(h)\right\|_{L^{2}(\Omega)}^{2}|| y|\nabla U|\left\|_{L^{\infty}(\Omega \times(0, \infty))}^{\frac{2 \beta}{\alpha}}\right\| y^{1-2 \alpha}|\nabla U| \|_{L^{\infty}(\Omega \times(0, \infty))}^{\frac{2(\alpha-\beta)}{\alpha}} \\
& \stackrel{(2.10),(2.14)}{\leq} C\left\|(-\Delta)^{\frac{\beta}{2}}(h)\right\|_{L^{2}(\Omega)}^{2}\left\|\left.g\right|_{L^{\infty}(\Omega)} ^{\frac{2 \beta}{\alpha}}\right\|(-\Delta)^{\alpha}(g) \|_{L^{\infty}(\Omega)}^{\frac{2(\alpha-\beta)}{\alpha}},
\end{aligned}
$$

which implies (2.9). The proof is complete.

\section{Proof of Theorem 1.2}

The case of the Fractional Laplacian in $\mathbb{R}^{d}$ is a straightforward modification of the previous arguments. We sketch it here for the reader's convenience. First we introduce the appropriate extensions, modified to suit our operator. Let $U, V, W$ be the solutions given by Theorem 1.4 of the boundary value problems:

$$
\begin{aligned}
& \begin{cases}\operatorname{div}\left(y^{1-2 \alpha} \nabla U\right)=0 & \text { in } \mathbb{R}^{d} \times(0, \infty), \\
U(x, 0)=g(x) & \text { in } \mathbb{R}^{d},\end{cases} \\
& \begin{cases}\operatorname{div}\left(y^{1-2 \alpha} \nabla V\right)=0 & \text { in } \mathbb{R}^{d} \times(0, \infty), \\
V(x, 0)=h(x) & \text { in } \mathbb{R}^{d},\end{cases} \\
& \begin{cases}\operatorname{div}\left(y^{1-2 \alpha} \nabla W\right)=0 & \text { in } \mathbb{R}^{d} \times(0, \infty), \\
W(x, 0)=g(x) h(x) & \text { in } \mathbb{R}^{d},\end{cases}
\end{aligned}
$$

Defining as before $Z=W-U V$, we have by construction

$$
-\lim _{y \rightarrow 0^{+}} y^{1-2 \alpha} Z_{y}(x, y)=\left[(-\Delta)^{\alpha}(g h)(x)-g(x)(-\Delta)^{\alpha}(h)(x)-h(x)(-\Delta)^{\alpha}(g)(x)\right]
$$

and $Z$ solves the extended problem

$$
\begin{cases}\left.\operatorname{div}\left(y^{1-2 \alpha} \nabla Z\right)\right)=-2 y^{1-2 \alpha} \nabla U \cdot \nabla V & \text { in } \mathbb{R}^{d} \times(0, \infty), \\ Z=0 & \text { on } \mathbb{R}^{d},\end{cases}
$$

Multiplying (3.4) by check this $\varphi=y^{-2 \alpha} Z$ and integrating by parts leads

$$
\begin{aligned}
& \frac{1}{4 \alpha} \int_{\mathbb{R}^{d}}\left|\lim _{y \rightarrow 0} y^{1-2 \alpha} Z_{y}(x, y)\right|^{2} d x+\int_{0}^{\infty} \int_{\mathbb{R}^{d}} y\left|\nabla\left(y^{-2 \alpha} Z\right)\right|^{2} d x d y \\
& \quad=2 \int_{0}^{\infty} \int_{\mathbb{R}^{d}} y^{1-2 \alpha} \nabla U \cdot \nabla V y^{-2 \alpha} Z d x d y .
\end{aligned}
$$

Using Hölder's inequality as before,

$$
\int_{\mathbb{R}^{d}}\left|\lim _{y \rightarrow 0} y^{1-2 \alpha} Z_{y}(x, y)\right|^{2} d x
$$




$$
\begin{aligned}
\leq & C\left\|y^{1-2 \alpha}|\nabla U|\right\|_{L^{\infty}\left(\mathbb{R}^{d} \times(0, \infty)\right)} \int_{0}^{\infty} \int_{\mathbb{R}^{d}}|\nabla V| y^{-2 \alpha}|Z| d x d y \\
\leq & C\left(\int_{0}^{\infty} \int_{\mathbb{R}^{d}} y^{1-2 \beta}|\nabla V|^{2} d x d y\right)^{1 / 2}\left(\int_{0}^{\infty} \int_{\mathbb{R}^{d}} y^{-1-2(2 \alpha-\beta)}|Z|^{2} d x d y\right)^{1 / 2} \\
& \times\left\|y^{1-2 \alpha}|\nabla U|\right\|_{L^{\infty}\left((0, \infty) \times \mathbb{R}^{d}\right)} .
\end{aligned}
$$

We just need to show then

$$
\begin{gathered}
\int_{0}^{\infty} \int_{\mathbb{R}^{d}} y^{1-2 \beta}|\nabla V|^{2} d x d y \leq C \int_{\mathbb{R}^{d}}\left|(-\Delta)^{\frac{\beta}{2}} h\right|^{2} d x \\
\int_{0}^{\infty} \int_{\mathbb{R}^{d}} y^{-1-2 \alpha}|Z|^{2} d x d y \leq C||(-\Delta)^{\frac{\beta}{2}}(h)\left\|_{L^{2}\left(\mathbb{R}^{d}\right)}^{2}\right\| g\left\|_{L^{\infty}\left(\mathbb{R}^{d}\right)}^{\frac{2 \beta}{\alpha}}\right\|(-\Delta)^{\alpha}(g) \|_{L^{\infty}\left(\mathbb{R}^{d}\right)}^{\frac{2(\alpha-\beta)}{\alpha}},
\end{gathered}
$$

and

$$
\left\|y^{1-2 \alpha}|\nabla U|\right\|_{L^{\infty}\left(\mathbb{R}^{d} \times(0, \infty)\right)} \leq C\left\|(-\Delta)^{\alpha}(g)\right\|_{L^{\infty}\left(\mathbb{R}^{d}\right)} .
$$

Compared to the previous section, the only point which is different is the one to prove (3.7). Here we just invoke the explicit expression of the Poisson kernel $P_{\alpha}$ which is (up to a universal normalizing constant)

$$
P_{\alpha}(x, y)=\frac{y^{2 \alpha}}{\left(|x|^{2}+y^{2}\right)^{\frac{d+2 \alpha}{2}}}
$$

and by construction, the solution $U$ which is the convolution of $P_{\alpha}$ with $g$ satisfies the desired estimates.

\section{APPENDIX}

In this appendix, we provide two results supporting our conjecture on the failure of the usual form of the Leibniz rule in the case of the restricted Laplacian. Our purpose is to relate a weighted (by a suitable power of the distance function) $L^{p}$ norm for $p=1,2$ of the function to the $L^{p}$ norm of its fractional Laplacian. We would like to make in particular three comments:

- by the very definition of the restricted Laplacian, since the functions are supported on $\Omega$, the Leibniz rule reduces to estimate the integrals

$$
\int_{\mathbb{R}^{d}} \frac{(f(x)-f(y))(g(x)-g(y))}{|x-y|^{d+2 \alpha}} d y
$$

and since we are interested in estimating $L^{2}$ norms in $\Omega$, one is led to consider quantities of the type

$$
\int_{\Omega \times \Omega} \frac{(f(x)-f(y))(g(x)-g(y))}{|x-y|^{d+2 \alpha}} d x d y, \quad \int_{\Omega \times \Omega^{c}} \frac{(f(x)-f(y))(g(x)-g(y))}{|x-y|^{d+2 \alpha}} d x d y
$$

- it is by now well-known that smooth functions that are compactly supported in $\Omega$ and have finite $H^{\alpha}$ semi-norm, behave like $\operatorname{dist}(x, \partial \Omega)^{\alpha}$ close to the boundary of $\Omega$.

- finally, notice that there is two different ways to define a semi-norm (even in $\mathbb{R}^{d}$ ) in $\dot{W}^{\alpha, p}\left(\mathbb{R}^{d}\right)$, namely

$$
\int_{\mathbb{R}^{d} \times \mathbb{R}^{d}} \frac{|f(x)-f(y)|^{p}}{|x-y|^{d+p \alpha}} d x d y \text { and } \int_{\mathbb{R}^{d}}\left|(-\Delta)^{\alpha / 2} f(x)\right|^{p} d x .
$$


In the case of the whole space $\mathbb{R}^{d}$ and $p=2$, these latter norms are equivalent. Actually, according to [Ste70], depending on $p$, these spaces are ordered for every $\alpha \in(0,1)$ in bounded domains and they are still equivalent for $p=2$.

According to the previous remarks, if one seeks for a counter-example, one would need to understand how the $L^{2}$ norm in $\mathbb{R}^{d}$ of the commutator behaves with respect to its $L^{2}$ norm in $\Omega$. The following computations show that the boundary behaviour plays a crucial role.

Let $\alpha \in(0,1 / 2)$. Let $u_{\varepsilon} \in C_{c}^{\infty}\left(B_{1}(0)\right)$ be a cut-off function such that $u_{\varepsilon}=1$ in $B_{1-2 \varepsilon}$, $u_{\varepsilon}=0$ in $B_{1-\varepsilon}^{c}$ and $\left|\nabla u_{\varepsilon}\right| \leq C \varepsilon, 0 \leq u_{\varepsilon} \leq 1$. We easily get first

$$
\int_{B_{1}} \frac{u_{\varepsilon}(x)^{2}}{\left(1-|x|^{2}\right)^{2 \alpha}} d x \sim 1, \quad \forall \varepsilon \in(0,1 / 10) .
$$

and for any $0<\alpha<\alpha_{0}<1 / 2$

$$
\int_{B_{1}} \int_{B_{1}} \frac{\left|u_{\varepsilon}(x)-u_{\varepsilon}(y)\right|^{2}}{|x-y|^{d+2 \alpha}} d x d y \lesssim_{s_{0}} \varepsilon^{1-2 \alpha_{0}} .
$$

Indeed,

$$
\begin{aligned}
& \int_{B_{1}} \int_{B_{1}} \frac{\left|u_{\varepsilon}(x)-u_{\varepsilon}(y)\right|^{2}}{|x-y|^{d+2 \alpha}} d x d y \\
& =2 \int_{B_{1} \backslash B_{1-3 \varepsilon}} \int_{B_{1} \backslash B_{1-3 \varepsilon}} \frac{\left|u_{\varepsilon}(x)-u_{\varepsilon}(y)\right|^{2}}{|x-y|^{d+2 \alpha}} d x d y+2 \int_{B_{1} \backslash B_{1-2 \varepsilon}} \int_{B_{1-3 \varepsilon}} \frac{\left|1-u_{\varepsilon}(y)\right|^{2}}{|x-y|^{d+2 \alpha}} d x d y \\
& \lesssim \varepsilon^{-2 \alpha_{0}} \int_{B_{1} \backslash B_{1-3 \varepsilon}} \int_{B_{1} \backslash B_{1-3 \varepsilon}} \frac{1}{|x-y|^{d-2\left(\alpha_{0}-\alpha\right)}} d x d y+\int_{B_{1} \backslash B_{1-2 \varepsilon}} \int_{B_{1-3 \varepsilon}} \frac{1}{|x-y|^{d+2 \alpha}} d x d y \\
& \lesssim \varepsilon^{-2 \alpha_{0}} \int_{B_{1} \backslash B_{1-3 \varepsilon}} d y+\int_{B_{1} \backslash B_{1-2 \varepsilon}} \varepsilon^{-2 \alpha} d y \\
& \lesssim \varepsilon^{1-2 \alpha_{0}}+\varepsilon^{1-2 \alpha} \lesssim \varepsilon^{1-2 \alpha_{0}} .
\end{aligned}
$$

On the other hand, for any $x \in B_{1}$

$$
\left\|(-\Delta)^{\alpha / 2} u_{\varepsilon}\right\|_{L^{2}\left(B_{1}\right)} \sim 1 .
$$

Indeed, for $x \in B_{1}$

$$
\begin{aligned}
\left|(-\Delta)^{\alpha / 2} u_{\varepsilon}(x)-\int_{B_{1}} \frac{u_{\varepsilon}(x)-u_{\varepsilon}(y)}{|x-y|^{d+\alpha}} d y\right| & =\left|\int_{B_{1}^{c}} \frac{u_{\varepsilon}(x)}{|x-y|^{d+\alpha}} d y\right| \\
& \lesssim \frac{\left|u_{\varepsilon}(x)\right|}{\left(1-|x|^{2}\right)^{\alpha}} .
\end{aligned}
$$

So,

$$
\begin{aligned}
\int_{B_{1}}\left|(-\Delta)^{\alpha / 2} u_{\varepsilon}(x)-\int_{B_{1}} \frac{u_{\varepsilon}(x)-u_{\varepsilon}(y)}{|x-y|^{d+\alpha}} d y\right|^{2} d x & =\left|\int_{B_{1}^{c}} \frac{u_{\varepsilon}(x)}{|x-y|^{d+\alpha}} d y\right| \\
& \lesssim \int_{B_{1}} \frac{\left|u_{\varepsilon}(x)\right|^{2}}{\left(1-|x|^{2}\right)^{2 \alpha}} d x \sim 1 .
\end{aligned}
$$

Moreover, for $\alpha<\alpha_{1}<\alpha_{2}<1 / 2$

$$
\int_{B_{1}}\left|\int_{B_{1}} \frac{u_{\varepsilon}(x)-u_{\varepsilon}(y)}{|x-y|^{d+\alpha}} d y\right|^{2} d x
$$




$$
\begin{aligned}
& \lesssim \alpha_{1} \int_{B_{1}} \int_{B_{1}} \frac{\left|u_{\varepsilon}(x)-u_{\varepsilon}(y)\right|^{2}}{|x-y|^{d+2 \alpha_{1}}} d y d x \\
& \stackrel{(2.5)}{\lesssim \alpha_{1}, \alpha_{2}} \varepsilon^{1-2 \alpha_{2} .}
\end{aligned}
$$

Combining this with (3.11) yields (3.10). As a consequence, we have

$$
\int_{B_{1}} \frac{u_{\varepsilon}(x)^{2}}{\left(1-|x|^{2}\right)^{2 \alpha}} d x \sim\left\|(-\Delta)^{\alpha / 2} u_{\varepsilon}\right\|_{L^{2}\left(B_{1}\right)}^{2} \sim 1
$$

but

$$
\int_{B_{1}} \int_{B_{1}} \frac{\left|u_{\varepsilon}(x)-u_{\varepsilon}(y)\right|^{2}}{|x-y|^{d+2 \alpha}} d x d y \rightarrow 0 \text { as } \varepsilon \rightarrow 0 .
$$

This is an explicit example that in a bounded domain for $\alpha<1 / 2$, the Hardy inequality in $L^{2}$ does not hold.

However, the analogous result in $L^{1}$ does hold:

Theorem 3.1. Let $u \in C_{c}^{\infty}(\Omega)$ be a solution to

$$
\int_{\mathbb{R}^{d}} \frac{u(x)-u(y)}{|x-y|^{d+2 \alpha}} d y=f(x)
$$

in $\Omega$, where $\Omega$ is smooth bounded domain. Then,

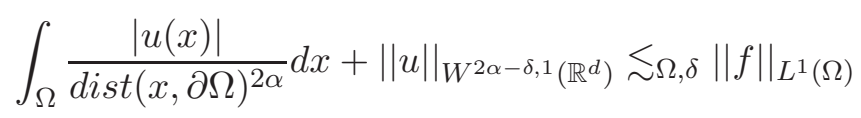

for any $\delta \in(0, \alpha / 4)$

Proof. Using $T_{\varepsilon}(u(x))=\operatorname{sign}(u(x)) \min \{\varepsilon,|u(x)|\}$ as test function,

$$
\int_{\mathbb{R}^{d}} \int_{\mathbb{R}^{d}} \frac{|u(x)-u(y)|\left|T_{\varepsilon}(u(x))-T_{\varepsilon}(u(y))\right|}{|x-y|^{d+2 \alpha}} d x d y=2 \int T_{\varepsilon}(u(x)) f(x) d x .
$$

This implies

$\int_{\Omega^{c}} \int_{\Omega} \frac{|u(x)|\left|T_{\varepsilon}(u(x))\right|}{|x-y|^{d+2 \alpha}} d x d y=\int_{\Omega^{c}} \int_{\Omega} \frac{|u(x)-u(y)|\left|T_{\varepsilon}(u(x))-T_{\varepsilon}(u(y))\right|}{|x-y|^{d+2 \alpha}} d x d y \leq \varepsilon \mid f \|_{L^{1}(\Omega)}$. So,

$$
\int_{\Omega^{c}} \int_{\Omega} \frac{|u(x)| \varepsilon^{-1} T_{\varepsilon}(|u(x)|)}{|x-y|^{d+2 \alpha}} d x d y \leq \mid f \|_{L^{1}(\Omega)} .
$$

Letting $\varepsilon \rightarrow 0$ to get

Since

$$
\int_{\Omega^{c}} \int_{\Omega} \frac{|u(x)|}{|x-y|^{d+2 \alpha}} d x d y \leq\|f\|_{L^{1}(\Omega)} .
$$

so,

$$
\int_{\Omega^{c}} \frac{1}{|x-y|^{d+2 \alpha}} d y \sim \operatorname{dist}(x, \partial \Omega)^{-2 \alpha}
$$

$$
\int_{\Omega} \frac{|u(x)|}{\operatorname{dist}(x, \partial \Omega)^{2 \alpha}} d x \lesssim\|f\|_{L^{1}(\Omega)}
$$

Moreover, we can write

$$
\int_{\mathbb{R}^{d}} \frac{u(x)-u(y)}{|x-y|^{d+2 \alpha}} d y=\mathbf{1}_{x \in \Omega} f(x)+\mathbf{1}_{x \notin \Omega} \int_{\Omega} \frac{-u(y)}{|x-y|^{d+2 \alpha}} d y:=g(x),
$$


for any $x \in \mathbb{R}^{d}$. Thus, by the standard regularity theory, one has

$$
\|u\|_{W^{2 \alpha-\delta, 1}\left(\mathbb{R}^{d}\right)} \lesssim \Omega, \delta\|g\|_{L^{1}\left(\mathbb{R}^{d}\right)}
$$

for any $\delta \in(0, \alpha / 2)$. Since

$$
\begin{aligned}
& \|g\|_{L^{1}\left(\mathbb{R}^{d}\right)} \leq\|f\|_{L^{1}(\Omega)}+\int_{\Omega^{c}} \int_{\Omega} \frac{|u(y)|}{|x-y|^{d+2 \alpha}} d y d x \\
& \lesssim\|f\|_{L^{1}(\Omega)}+\int_{\Omega} \frac{|u(y)|}{\operatorname{dist}(y, \partial \Omega)^{2 \alpha}} d y \\
& \text { (3.15) } \\
& \lesssim\|f\|_{L^{1}(\Omega)},
\end{aligned}
$$

we get (3.13).

ACKNOWLEDGments. Quoc-Hung Nguyen is supported by the Shanghai Tech University startup fund. J.L.V. partially funded by Project PGC2018-098440-B-I00 from MICINN, of the Spanish Government. Partially performed as an Honorary Professor at Univ. Complutense de Madrid.

\section{REFERENCES}

[BSS20a] Denis Brazke, Armin Schikorra, and Yannick Sire. In preparation, 2020.

[BSS20b] Denis Brazke, Armin Schikorra, and Yannick Sire. Characterization of bmo via carleson measures on riemannian manifolds. To appear IMRN, 2020.

[BSV15] Matteo Bonforte, Yannick Sire, and Juan Luis Vázquez. Existence, uniqueness and asymptotic behaviour for fractional porous medium equations on bounded domains. Discrete Contin. Dyn. Syst., 35(12):5725-5767, 2015.

[BV16] Claudia Bucur and Enrico Valdinoci. Nonlocal diffusion and applications, volume 20 of Lecture Notes of the Unione Matematica Italiana. Springer, [Cham]; Unione Matematica Italiana, Bologna, 2016.

[CDDS11] Antonio Capella, Juan Dávila, Louis Dupaigne, and Yannick Sire. Regularity of radial extremal solutions for some non-local semilinear equations. Comm. Partial Differential Equations, 36(8):1353-1384, 2011.

$\left[\mathrm{CdPF}^{+} 17\right]$ José Antonio Carrillo, Manuel del Pino, Alessio Figalli, Giuseppe Mingione, and Juan Luis Vázquez. Nonlocal and nonlinear diffusions and interactions: new methods and directions, volume 2186 of Lecture Notes in Mathematics. Springer, Cham; Fondazione C.I.M.E., Florence, 2017. Lectures from the CIME Course held in Cetraro, July 4-8, 2016, Edited by Matteo Bonforte and Gabriele Grillo, Fondazione CIME/CIME Foundation Subseries.

[CI16] Peter Constantin and Mihaela Ignatova. Critical SQG in bounded domains. Ann. PDE, 2(2):Art. 8, 42, 2016.

[CS05] Zhen-Qing Chen and Renming Song. Two-sided eigenvalue estimates for subordinate processes in domains. J. Funct. Anal., 226(1):90-113, 2005.

[CS07] Luis Caffarelli and Luis Silvestre. An extension problem related to the fractional Laplacian. Comm. Partial Differential Equations, 32(7-9):1245-1260, 2007.

[CT10] Xavier Cabré and Jinggang Tan. Positive solutions of nonlinear problems involving the square root of the Laplacian. Adv. Math., 224(5):2052-2093, 2010.

[CY17] Sun Yung Alice Chang and Ray A. Yang. On a class of non-local operators in conformal geometry. Chin. Ann. Math. Ser. B, 38(1):215-234, 2017.

[Dav90] E. B. Davies. Heat kernels and spectral theory, volume 92 of Cambridge Tracts in Mathematics. Cambridge University Press, Cambridge, 1990.

[Fre13] Dorothee Frey. Paraproducts via $H^{\infty}$-functional calculus. Rev. Mat. Iberoam., 29(2):635-663, 2013. 
[KP88] Tosio Kato and Gustavo Ponce. Commutator estimates and the Euler and Navier-Stokes equations. Comm. Pure Appl. Math., 41(7):891-907, 1988.

[KPV93] Carlos E. Kenig, Gustavo Ponce, and Luis Vega. Well-posedness and scattering results for the generalized Korteweg-de Vries equation via the contraction principle. Comm. Pure Appl. Math., 46(4):527-620, 1993.

[KSV19] Panki Kim, Renming Song, and Zoran Vondraček. Potential theory of subordinate killed Brownian motion. Trans. Amer. Math. Soc., 371(6):3917-3969, 2019.

[KSV20] Panki Kim, Renming Song, and Zoran Vondraček. On the boundary theory of subordinate killed Lévy processes. Potential Anal., 53(1):131-181, 2020.

[Li19] Dong Li. On Kato-Ponce and fractional Leibniz. Rev. Mat. Iberoam., 35(1):23-100, 2019.

[LS20] Enno Lenzmann and Armin Schikorra. Sharp commutator estimates via harmonic extensions. Nonlinear Anal., 193:111375, 2020.

[LS21] Dong Li and Yannick Sire. Higher order kato-ponce estimates and counter-examples. In preparation, 2021.

[MN14] Roberta Musina and Alexander I. Nazarov. On fractional Laplacians. Comm. Partial Differential Equations, 39(9):1780-1790, 2014.

[NV18] Quoc-Hung Nguyen and Juan-Luis Vazquez. Porous medium equation with nonlocal pressure in a bounded domain. Com PDEs., 43:1502-1539, 2018.

[ST10] Pablo Raúl Stinga and José Luis Torrea. Extension problem and Harnack's inequality for some fractional operators. Comm. Partial Differential Equations, 35(11):2092-2122, 2010.

[Ste70] Elias M. Stein. Singular integrals and differentiability properties of functions. Princeton Mathematical Series, No. 30. Princeton University Press, Princeton, N.J., 1970.

[SV14] Raffaella Servadei and Enrico Valdinoci. On the spectrum of two different fractional operators. Proc. Roy. Soc. Edinburgh Sect. A, 144(4):831-855, 2014.

[Zyg59] A. Zygmund. Trigonometric series. 2nd ed. Vols. I, II. Cambridge University Press, New York, 1959 .

2010 Mathematics Subject Classification. Primary: 42B37. Secondary: 35J25

Keywords: Fractional Laplacian operators on domains, commutator estimates, Leibniz rule.

Shanghaitech University, 393 Middle Huaxia Road, Pudong, Shanghai, 201210, China

Email address: qhnguyen@shanghaitech.edu.cn

Department of Mathematics, Johns Hopkins University, Baltimore, MD 21218, United States

Email address: ysire1@jhu.edu

Departamento de Matemáticas, Universidad Autónoma de Madrid, 28049 Madrid, Spain

Email address: juanluis.vazquez@uam.es 